\title{
Significant value of autopsy for quality management in cardiac surgery
}

\author{
Ardawan J. Rastan, MD, ${ }^{a}$ Jan F. Gummert, MD, PhD, ${ }^{a}$ Nicole Lachmann, ${ }^{\text {a }}$ Thomas Walther, MD, PhD, ${ }^{\text {a }}$ \\ Dierck V. Schmitt, MD, ${ }^{a}$ Volkmar Falk, MD, PhD, ${ }^{a}$ Nico Doll, MD, ${ }^{a}$ Paul Caffier, MD, ${ }^{b}$ Markus M. Richter, MD, \\ Christian Wittekind, MD, PhD, and Friedrich W. Mohr, MD, PhDa
}

Objective: With recent advances in diagnostic imaging, the value of autopsy has been called into question. The aim of our study was to assess the current impact of autopsy for early postoperative quality management in cardiac surgery.

Methods: Between 2000 and 2003, a total of 14,313 patients underwent cardiac surgery at our center. Of these, 898 patients $(6.3 \%)$ died, and autopsy was performed in 468 cases $(52.1 \%)$. Data from clinical and postmortem examination were prospectively analyzed regarding causes of death, postoperative complications, concomitant diseases, and surgery-associated pathologic findings.

Results: Mean age was 68.7 years. Mean survival was 13.9 postoperative days. On autopsy, causes of death were cardiac in $49.8 \%$ of cases $(n=233)$, respiratory in $8.3 \%(\mathrm{n}=39)$, cerebral in $6.4 \%(\mathrm{n}=30)$, abdominal in $4.7 \%(\mathrm{n}=22)$, multiorgan failure or sepsis in $14.9 \%(\mathrm{n}=70)$, pulmonary embolism in $6.6 \%(\mathrm{n}=31)$, procedure associated in $8.3 \%(\mathrm{n}=39)$, and others in $0.9 \%(\mathrm{n}=4)$. Discrepancies between clinical and postmortem determinations of cause of death were found in 108 cases $(23.1 \%)$. These were acute myocardial infarction $(\mathrm{n}=38)$, low cardiac output $(n=9)$, respiratory $(n=8)$, cerebral $(n=5)$, abdominal $(n=7)$, multiorgan failure or sepsis $(\mathrm{n}=12)$, pulmonary embolism $(\mathrm{n}=18)$, and procedure associated (11). Clinically unrecognized postoperative complications were found in 364 cases (77.8\%). Unknown concomitant diseases were found in 464 cases $(99.1 \%)$, with potential therapeutic relevance in 90 cases $(19.2 \%)$. In 85 cases $(18.2 \%)$, autopsy examination revealed 96 premortem unrecognized surgery-associated pathologic findings.

From the Department of Cardiac Surgery, Heart Center, ${ }^{a}$ and the Institute of Pathology, ${ }^{\text {b }}$ University of Leipzig, Leipzig, Germany.

Read at the Eighty-fourth Annual Meeting of The American Association for Thoracic Surgery, Toronto, Ontario, Canada, April 25-28, 2004.

Received for publication May 15, 2004; revisions received Dec 4, 2004; accepted for publication Dec 10, 2004.

Address for reprints: Ardawan Julian Rastan, MD, Department of Cardiac Surgery, Heart Center Leipzig, Struempellstr. 39, 04289 Leipzig, Germany (E-mail address: rastan@rz.uni-leipzig.de).

J Thorac Cardiovasc Surg 2005;129:1292-300 $0022-5223 / \$ 30.00$

Copyright ( $(2) 2005$ by The American Association for Thoracic Surgery

doi:10.1016/j.jtcvs.2004.12.018
Conclusion: A high overall discrepancy rate between premortem and autopsy diagnoses was recognized. Autopsy revealed clinically relevant information in a significant number of cases. Therefore autopsy remains essential for quality assessment in perioperative treatment.

$\mathrm{T}$ o reduce unfavorable patient outcomes and to learn from previous misjudgments, detailed information should be available regarding patients who die in the early postoperative course after cardiac surgery to reflect individual surgical indication and analyze surgical and postoperative treatment. This includes an accurate determination of cause of death and critical recognition of perioperative complications. Both can be achieved by autopsy examination and subsequent postmortem evaluation. ${ }^{1-7}$ In the past, autopsy has been the widely accepted criterion standard for the evaluation of causes of in-hospital death. This is in contrast to a dramatic fall in autopsy rate in most countries in recent years. The aim of this study was to prospectively assess the current value of autopsy examination as an instrument for quality management in cardiac surgery. We compared clinical and autopsy findings concerning the confirmation of causes of death and the recognition 
of perioperative complications. In addition, the incidences of clinically unknown concomitant diseases, premortem unexpected technical failures, and surgery-associated pathologic findings were evaluated.

\section{Material and Methods}

\section{Patients and Procedures}

Between January 2000 and December 2003, there were 898 postoperative deaths, resulting in an overall in-hospital mortality of $6.3 \%$ and a corresponding 30-day mortality of 5.4\%. Informed consent was obtained for 468 patients (52.1\%), forming the study population. Autopsy was performed for clinicopathologic reasons in all cases.

\section{Study Protocol}

Clinical data for all in-hospital deaths after cardiac surgery were prospectively recorded. Clinical causes of death were determined by the intensive care unit (ICU) physicians, taking into consideration available clinical data, the postoperative ICU course, and specific surgical aspects. A detailed hospital report, including the clinical cause of death, perioperative complications, and all known concomitant diseases, was appended to the certificate of death and sent to the pathologist.

Autopsy examination was performed on average $2.9 \pm 1.5$ days after death (range 1-8 days) through standard incisions. Each patient underwent standard sampling of the coronary arteries and the myocardium, as well as any other relevant organ. At minimum four sections of ventricular myocardium were taken, and acute myocardial infarction (AMI) was diagnosed by hematoxylin and eosin staining. A clinically based case summary was presented by the surgeon at the regular mortality and morbidity conference, followed by a detailed presentation of autopsy findings by the pathologist, including autopsy-determined cause of death. Therefore all clinical data were included to find an autopsy-based cause of death in accordance with the clinicians. Additional notes from the postmortem reports were analyzed and compared with clinical data concerning postoperative complications, as well as concomitant diseases, noting agreements or contradictions. Premortem known and unexpected pathologies of the surgical situs were also recognized. Multiple preoperative and postoperative variables were recorded (Table 1).

\section{Definitions}

Cardiac cause of death was defined clinically as attributable to acute heart failure (AHF, low cardiac output syndrome), with or without signs of acute AMI or sudden cardiac death (SCD). AHF without AMI was defined as failure of at least one ventricle resulting in pulmonary edema, acidosis, hypotension, and consecutive organ failure, including valve dysfunction and arrhythmias. AHF was confirmed by autopsy in the presence of acute dilated ventricles, pulmonary edema, and secondary organ damage but no evidence for another primary cause of death. AHF with AMI was assumed as the cause of death in the presence of AMI large enough to account for low cardiac output syndrome. SCD was defined as immediate, unexpected death within 1 hour as a result of cardiac problems or in patients
TABLE 1. Patient characteristics, surgical procedures, and major postoperative treatment

\begin{tabular}{|c|c|c|}
\hline & $\begin{array}{l}\text { Autopsy patients } \\
\quad(\mathrm{n}=468)\end{array}$ & $\begin{array}{c}\text { Nonautopsy } \\
\text { patients } \\
(n=430)\end{array}$ \\
\hline Age (y) & $68.7 \pm 11.3$ & $70.5 \pm 9.5$ \\
\hline Male $(\%)$ & $65.2 \%$ & $62.3 \%$ \\
\hline Coronary artery disease (\%) & $63.9 \%$ & $65 \%$ \\
\hline History of AMI (\%) & $33.1 \%$ & $33.6 \%$ \\
\hline Diabetes (\%) & $40.6 \%$ & $39.3 \%$ \\
\hline Arterial hypertension (\%) & $70.5 \%$ & $73.2 \%$ \\
\hline Body mass index $\left(\mathrm{kg} / \mathrm{m}^{2}\right)$ & $30.1 \pm 5.0$ & $29.6 \pm 6.3$ \\
\hline Additive EuroSCORE & $11.4 \pm 4.3$ & $11.7 \pm 3.9$ \\
\hline Log EuroSCORE & $32.4 \pm 24.2$ & $31.9 \pm 22.8$ \\
\hline Ejection fraction $(\%)$ & $38.5 \% \pm 16.3 \%$ & $40.9 \% \pm 18.4 \%$ \\
\hline Emergency indication (\%) & $32.9 \%$ & $33.5 \%$ \\
\hline Urgent indication $(\%)$ & $22 \%$ & $22.8 \%$ \\
\hline $\begin{array}{l}\text { Standalone } \mathrm{CABG} \\
\text { procedure }(\%)\end{array}$ & $49.1 \%$ & $51.4 \%$ \\
\hline Aortic valve surgery (\%) & $10.2 \%$ & $10.0 \%$ \\
\hline Mitral valve surgery (\%) & $7.2 \%$ & $7.0 \%$ \\
\hline CABG with valve surgery $(\%)$ & $9.2 \%$ & $8.8 \%$ \\
\hline Reoperation (\%) & $22.2 \%$ & $19.3 \%$ \\
\hline $\begin{array}{l}\text { Preoperative low cardiac } \\
\text { output }(\%)\end{array}$ & $27.6 \%$ & $25.4 \%$ \\
\hline Preoperative IABP (\%) & 11.3 & 14.9 \\
\hline Perioperative IABP (\%) & 24.8 & 22.8 \\
\hline Postoperative hemodialysis (\%) & 54.9 & 57.2 \\
\hline Perioperative ECLS (\%) & 8.8 & 9.8 \\
\hline Postoperative laparotomy (\%) & 12.4 & 12.1 \\
\hline Rethoracotomy (\%) & 43.6 & 50.1 \\
\hline Postoperative survival (d) & $13.9(0-122)$ & $15.3(0-141)$ \\
\hline
\end{tabular}

$I A B P$, Intra-aortic balloon pump; ECLS, extracorporeal life support. $P$ was not significant for all differences between groups. Values are given as mean $\pm S D$ or mean and range.

found dead on the regular ward after an uneventful postoperative course.

Nonseptic and septic multiorgan failure (MOF) were defined in accordance to the definition of the American College of Chest Physicians and Society of Critical Care Medicine Consensus Conference. ${ }^{8}$ MOF was assessed in patients with primary organ failure of at least two organ systems and corresponding morphologic findings on the condition that no single organ damage could be identified as predominant cause of death. MOF was not assessed in patients with secondary organ damage caused by ischemia or hypoxia as a result of cardiac or pulmonary failure. Fatal pulmonary embolism (PE) was presumed to be cause of death in patients with obliteration of at least two lobular pulmonary arteries with typical clinical findings and in the absence of evidence for another cause of death.

\section{Statistics}

Data were $100 \%$ complete for premortem and postmortem records. Results are expressed as mean \pm SEM. Data were compared with the $\chi^{2}$ test or 2-tailed Student $t$ test as appropriate. SPSS 11.0 
TABLE 2. Confirmation and contradiction of clinical causes of death by autopsy findings

\begin{tabular}{|c|c|c|c|c|c|c|c|c|c|}
\hline \multirow[b]{2}{*}{ Cause of death } & \multicolumn{2}{|c|}{ Clinical } & \multicolumn{2}{|c|}{ Autopsy } & \multicolumn{2}{|c|}{ Confirmation } & \multicolumn{2}{|c|}{ Contradiction } & \multirow{2}{*}{$\begin{array}{c}\text { Sensitivity } \\
(\%)\end{array}$} \\
\hline & No. & $\%$ & No. & $\%$ & No. & $\%$ & No. & $\%$ & \\
\hline Cardiac & 220 & 47.0 & 233 & 49.8 & 186 & 84.5 & 34 & 15.5 & 79.8 \\
\hline AHF with AMI & 112 & 23.9 & 163 & 34.8 & 95 & 84.8 & 17 & 15.2 & 58.3 \\
\hline AHF without AMI & 76 & 16.3 & 62 & 13.3 & 43 & 56.6 & 33 & 43.4 & 69.4 \\
\hline SCD & 32 & 6.8 & 8 & 1.7 & 8 & 25.0 & 24 & 75.0 & 100.0 \\
\hline Respiratory & 57 & 12.2 & 39 & 8.3 & 31 & 54.4 & 26 & 45.6 & 79.5 \\
\hline ARDS & 8 & 1.7 & 17 & 3.6 & 5 & 62.5 & 3 & 37.5 & 29.4 \\
\hline Pneumonia & 44 & 9.4 & 17 & 3.6 & 14 & 31.8 & 30 & 68.2 & 82.3 \\
\hline Lung bleeding & 5 & 1.1 & 5 & 1.1 & 2 & 40.0 & 3 & 60.0 & 40.0 \\
\hline Cerebral & 29 & 6.2 & 30 & 6.4 & 25 & 86.2 & 4 & 13.8 & 83.3 \\
\hline Stroke & 17 & 3.6 & 18 & 3.8 & 13 & 76.5 & 4 & 23.5 & 72.2 \\
\hline Bleeding & 5 & 1.1 & 5 & 1.1 & 5 & 100.0 & 0 & 0 & 100.0 \\
\hline Primary edema & 6 & 1.3 & 6 & 1.3 & 6 & 100.0 & 0 & 0 & 100.0 \\
\hline Cerebral embolism & 1 & 0.2 & 1 & 0.2 & 1 & 100.0 & 0 & 0 & 100.0 \\
\hline Abdominal & 23 & 4.9 & 22 & 4.7 & 15 & 65.2 & 8 & 34.8 & 68.2 \\
\hline Intestinal ischemia & 13 & 2.8 & 11 & 2.3 & 9 & 69.2 & 4 & 30.8 & 81.2 \\
\hline Intestinal perforation & 6 & 1.3 & 6 & 1.3 & 4 & 66.6 & 2 & 33.3 & 66.6 \\
\hline Pancreatitis & 1 & 0.2 & 4 & 0.9 & 1 & 100.0 & 0 & 0 & 25.0 \\
\hline Liver failure & 2 & 0.4 & 1 & 0.2 & 1 & 50.0 & 1 & 50.0 & 100.0 \\
\hline Others & 1 & 0.2 & 0 & 0 & 0 & 0 & 1 & 100.0 & \\
\hline MOF & 88 & 18.8 & 70 & 14.9 & 58 & 65.9 & 30 & 34.1 & 82.8 \\
\hline Septic & 51 & 10.9 & 42 & 9.0 & 37 & 72.5 & 14 & 27.5 & 88.1 \\
\hline Nonseptic & 37 & 7.9 & 28 & 6.0 & 21 & 56.7 & 16 & 43.2 & 75.0 \\
\hline PE & 16 & 3.4 & 31 & 6.6 & 13 & 81.3 & 3 & 18.7 & 41.9 \\
\hline Procedure associated & 29 & 6.2 & 39 & 8.3 & 28 & 96.6 & 1 & 3.4 & 71.8 \\
\hline Bleeding & 7 & 1.5 & 12 & 2.6 & 7 & 100.0 & 0 & 0 & 58.3 \\
\hline Aortic rupture & 4 & 0.9 & 5 & 1.1 & 4 & 100.0 & 0 & 0 & 80.0 \\
\hline Left ventricular rupture & 6 & 1.3 & 7 & 1.5 & 6 & 100.0 & 0 & 0 & 85.7 \\
\hline Tamponade & 1 & 0.2 & 4 & 0.9 & 1 & 100.0 & 0 & 0 & 25.0 \\
\hline Aortic dissection & 6 & 1.3 & 6 & 1.3 & 6 & 100.0 & 0 & 0 & 100.0 \\
\hline Mediastinitis & 5 & 1.1 & 5 & 1.1 & 4 & 80.0 & 1 & 20.0 & 80.0 \\
\hline Others & 6 & 1.3 & 4 & 0.9 & 4 & 66.7 & 2 & 33.3 & \\
\hline Total organ related & 468 & 100.0 & 468 & 100.0 & 360 & 76.9 & 108 & 23.1 & \\
\hline Total diagnosis related & 468 & 100.0 & 468 & 100.0 & 310 & 66.2 & 158 & 33.8 & \\
\hline
\end{tabular}

ARDS, Adult respiratory distress syndrome. Confirmation represents positive predictive value, autopsy-confirmed clinical cause of death related to all clinically assumed causes of death. Sensitivity represents correctly determined clinical cause of death related to causes of death recognized by autopsy examination.

(SPSS Inc, Chicago, Ill) and Microsoft Excel (Microsoft Corporation, Redmond, Wash) were used for statistical calculation. Validity of each clinical cause of death was expressed as positive predictive value. Clinical assessment of each cause of death was expressed as sensitivity.

\section{Results}

Overall in-hospital mortalities were $3.8 \%$ for elective procedures and $19.4 \%$ for emergency indications. Comparison of the patients who did and did not undergo autopsy revealed no significant differences (Table 1).

\section{Causes of Death}

Clinical and autopsy causes of death are shown in Table 2. On the basis of an organ-related evaluation, we found a confirmation by autopsy of the clinically assumed causes of death in $76.9 \%$ of cases. Confirmation was comparatively high for cardiac $(84.5 \%)$, cerebral $(86.2 \%)$, and procedureassociated causes of death $(96.6 \%)$ and for fatal PE $(81.3 \%)$. Confirmation was worse for clinically determined death related to respiratory $(54.4 \%)$ or abdominal $(65.2 \%)$ causes and for MOF (65.9\%).

Regarding diagnosis-related causes of death we found some significant discrepancies. Prediction of cardiac death from AMI was better than prediction of AHF without AMI or SCD. Clinically presumed respiratory causes of death were not confirmed by autopsy for a significant number of patients, $37.5 \%$ for adult respiratory distress syndrome and $68.2 \%$ for pneumonia. On the other hand, clinical assess- 
TABLE 3. Clinical and autopsy causes of death

\begin{tabular}{|c|c|c|c|c|c|c|c|c|c|c|c|c|c|c|}
\hline \multirow[b]{2}{*}{ Clinical } & \multicolumn{14}{|c|}{ Autopsy } \\
\hline & $\begin{array}{l}\text { AHF } \\
\text { with } \\
\text { AMI }\end{array}$ & $\begin{array}{c}\text { AHF, } \\
\text { no } \\
\text { AMI }\end{array}$ & SCD & ARDS & Pneumonia & $\begin{array}{c}\text { Lung } \\
\text { bleeding }\end{array}$ & Cerebral & Abdominal & $\begin{array}{c}\text { Septic } \\
\text { MOF }\end{array}$ & $\begin{array}{c}\text { Nonseptic } \\
\text { MOF }\end{array}$ & PE & Procedural & Others & Total \\
\hline AHF with AMI & 95 & 9 & & & & & 1 & 1 & & 1 & 4 & 1 & & 112 \\
\hline AHF, no AMI & 16 & 43 & & & 2 & 2 & 2 & 3 & 1 & 2 & 2 & 3 & & 76 \\
\hline SCD & 14 & 1 & 8 & & 1 & & & & & & 4 & 4 & & 32 \\
\hline ARDS & & & & 5 & & 1 & & 1 & 1 & & & & & 8 \\
\hline Pneumonia & 11 & 3 & & 9 & 14 & & 1 & & & 2 & 4 & & & 44 \\
\hline Lung bleeding & 1 & & & & & 2 & & & & 1 & 1 & & & 5 \\
\hline Cerebral & 1 & 2 & & & & & 25 & & & & 1 & & & 29 \\
\hline Abdominal & 4 & 1 & & & & & & 15 & 3 & & & & & 23 \\
\hline Septic MOF & 7 & 1 & & 3 & & & & 2 & 37 & & 1 & & & 51 \\
\hline Nonseptic MOF & 12 & 2 & & & & & 1 & & & 21 & & 1 & & 37 \\
\hline PE & & & & & & & & & & 1 & 13 & 2 & & 16 \\
\hline Procedural & & & & & & & & & & & 1 & 28 & & 29 \\
\hline Others & 2 & & & & & & & & & & & & 4 & 6 \\
\hline Total & 163 & 62 & 8 & 17 & 17 & 5 & 30 & 22 & 42 & 28 & 31 & 39 & 4 & 468 \\
\hline
\end{tabular}

$A H F$, Acute heart failure; $A R D S$, adult respiratory distress syndrome.

ments of almost all cerebral and procedure-associated deaths were appropriate. For diagnosis-related causes of death, overall confirmation between clinical and autopsy causes of death was only $66.2 \%$ (Table 2 ).

Cardiac causes of death were identified by autopsy in $49.8 \%$ of our cases but had been clinically presumed as cardiac in 186 cases only. This resulted in a clinical sensitivity of cardiac cause of death of $79.8 \%$. Regarding cardiac death from AMI, the sensitivity was reduced to $58.3 \%$. Clinical sensitivity was also low for adult respiratory distress syndrome $(29.4 \%)$, lung bleeding $(40.0 \%)$, pancreatitis (25.0\%), PE (41.9\%), and pericardial tamponade $(25.0 \%)$. It was high for cerebral and other procedure-associated causes of death and for SCD.

With respect to organ-related causes of death, we found unexpected deaths in 108 cases (23.1\%). With diagnosis-related analysis, discrepancies between clinical and autopsy causes of death became more evident, leading to 158 clinically unexpected causes of death $(33.8 \%)$. The main reason for this finding was that AMI could be identified by clinical assessment in only $58.3 \%$ of the patients (95/163; Table 3). Cause of death was assessed clinically in these patients as cardiac (low cardiac output without AMI or SCD; Table 3) or extracardiac, usually pneumonia and MOF. Other significant discrepancies were found in the respiratory system. Among patients with clinically presumed pneumonia, this cause of death was confirmed by autopsy for only $31.8 \%$. Contradictory autopsy causes of death in these patients were frequently AMI and adult respiratory distress syndrome.

Fatal PE was identified as cause of death in 31 cases $(6.6 \%)$. For 13 of these patients $(41.9 \%)$, the diagnosis had been clinically assumed; however, in 18 cases, PE could not be identified before death. Clinically assumed causes of death in these patients were predominantly AMI $(n=4), \operatorname{SCD}(n=4)$, and pneumonia $(n=4)$. In another 3 cases, PE was clinically diagnosed but could not be confirmed by autopsy. In 2 of these cases, technical failures were responsible for death (pericardial tamponade, coronary artery bypass grafting [CABG] bleeding).

A reason for SCD was found by autopsy in 24 of 32 cases (Table 3). Four procedure-associated reasons were $\mathrm{CABG}$ bleeding, left ventricular rupture, pericardial tamponade, and aortic rupture. In the remaining 8 cases, no decisive findings for death were found. These patients had unwitnessed SCD, and malignant arrhythmia had to be assumed. Six of these patients had undergone aortic valve surgery.

\section{Perioperative Complications}

A total of 783 perioperative complications were found in 364 of 468 patients at autopsy $(77.8 \%$, mean 2.2 complications/patient). Most $(\mathrm{n}=606,77.4 \%)$ were unknown before death (Figure 1). Pathologic findings included arterial embolism in 34 patients (52.9\% clinically undetected), deep venous thrombosis (DVT) of the upper extremity in 179 (96.6\%), DVT of the lower extremity in 63 (93.7\%), minor $\mathrm{PE}$ in 96 (91.7\%), pneumonia in 106 (44.3\%), acute splenic infarction in $87(83.9 \%)$, acute renal infarction in 57 $(93.9 \%)$, minor stroke in 52 (42.3\%), minor cerebral bleeding in 29 (79.3\%), intestinal ischemia in $37(62.2 \%)$, intestinal perforation in $18(72.2 \%)$, and acute pancreatitis in 25 $(56.0 \%)$. We found no significant relation between these pathologic findings and the surgical procedure performed, 
the length of postoperative survival, or the priority of the operation.

\section{Concomitant Diseases}

A total of 928 of the following diseases could be morphologically identified in 464 patients $(99.1 \%)$ by autopsy. Of these, 535 were unknown before death, representing 57.6\%. In detail, myocardial hypertrophy was found in 187 patients (36.4\% clinically undetected), occlusive arterial disease in 77 (48.1\% clinically undetected), former DVT in 30 (33.3\% clinically undetected), pulmonary emphysema in 77 (41.6\%), former PE in 27 (18.5\%), former stroke in 44 (31.8\%), renal cyst in 83 (88.0\%), liver degeneration in 53 $(90.6 \%)$, liver cirrhosis in $58(48.3 \%)$, bile stone disease in $51(78.4 \%)$, chronic pancreatitis in $14(85.7 \%)$, colon diverticulosis in $60(83.3 \%)$, benign tumors in 102 (78.3\%), and malignant tumors in $65(44.6 \%)$. Malignant tumors were lymphoma in 8 cases $(\mathrm{n}=3)$, lung carcinoma in $7(\mathrm{n}$ $=3)$, renal carcinoma in $5(\mathrm{n}=4)$, prostatic carcinoma in $15(\mathrm{n}=6)$, colorectal carcinoma in $9(\mathrm{n}=3)$, breast carcinoma in $4(\mathrm{n}=0)$, and others in $17(\mathrm{n}=10)$.

Unexpected occlusive artery disease $(\mathrm{n}=37)$, liver cirrhosis $(\mathrm{n}=28)$, and malignant tumors $(\mathrm{n}=29)$ were considered to be of relevance for surgical indication and therapy. These were found in 90 patients, representing $19.2 \%$ of the study population. Unexpected occlusive arterial disease was found in 19 of 130 (14.6\%) of emergency patients versus 18 of 338 (5.3\%) of nonemergency patients $(P<.001)$. Unknown liver cirrhosis was recognized in $13.8 \%$ of patients with emergency indications versus $3.0 \%$ with nonemergency indications $(P<.001)$. Incidences of premortem unrecognized malignant tumors were 6.9\% among emergency patients versus $5.9 \%$ among nonemergency patients $(P=.49)$.

\section{Technical Failures and Surgical Pathologic Findings}

In total, 96 premortem unknown surgery-associated pathologic findings were revealed by autopsy examination in 85 patients (18.2\%), including 45 patients $(9.6 \%)$ with significant $\mathrm{CABG}$ dysfunction. In addition, significant numbers of clinically undetected intracardiac $(1.2 \%)$ and prosthetic valve thrombi (3.6\%) were found. Other unexpected findings were bleeding $(1.1 \%)$, tamponade $(0.6 \%)$, prosthetic valve endocarditis $(0.4 \%)$, ostial right coronary artery stenosis after aortic valve replacement $(0.9 \%)$, unknown native coronary artery disease (1.5\%), left ventricular dissection $(0.2 \%)$, aortic rupture $(0.2 \%)$, mediastinitis $(0.2 \%)$, and others $(0.9 \%)$.

\section{Discussion}

In recent years, quality management in medicine has focused on the development of new instruments to improve medical services and allow internal and external quality control. Particularly in operative disciplines, these mechanisms are mandatory to guarantee modern and accurate surgical treatment. In parallel, scoring systems and risk stratifications have been developed, allowing a more meaningful comparison of surgical results. All such instruments urgently need extensive information about patient characteristics and perioperative outcome. Surprisingly, autopsy examination, although a proven instrument for quality assessment, has decreased dramatically in the past decades. ${ }^{4,5,9,10}$ One reason for this conflicting development could be the misconception that current diagnostic tools are highly accurate, making autopsies unnecessary. However, several studies disprove this factor by demonstrating consistent discrepancies between clinical and autopsy findings during different diagnostic eras. ${ }^{5,9-11}$

In the few published reports of postmortem findings after cardiac or thoracic surgery, the autopsy rate ranged from $24 \%$ to $88 \%$, significantly higher than autopsy rates reported for the average hospital population. ${ }^{12-18}$ Most of our patients dying after cardiac surgery had urgent or emergency surgical indications and were at high risk for unfavorable outcome, as expressed by increased preoperative risk score. Regarding patient age and postoperative survival, our findings are similar to previously published series in cardiac surgery (Table 4).

Regarding causes of death from other series, some discrepancies are evident. Obviously, the rate of cardiacrelated deaths in our patients was similar to findings by Lee and Gallagher ${ }^{13}$ but was higher than in the series reported by Zehr and associates ${ }^{14}$ and lower than in the series of Deiwick and associates ${ }^{12}$ and Goodwin and coworkers. ${ }^{15}$ Reasons might be the high proportion of death of unknown cause reported by Zehr and associates ${ }^{14}$ subtle differences in final autopsy assessment, differences in patient characteristics, and study population size. These could also be reasons for differences in proportion of deaths from abdominal and respiratory causes or surgery-associated causes of death.

In accordance with Barendregt and colleagues, ${ }^{3}$ we found a comparatively high confirmation of more than $80 \%$ for cardiac, cerebral, and procedure-associated causes of death. ${ }^{3}$ In addition to typical clinical symptoms, potential reasons for the good prediction of these causes of death might be such highly sensitive and specific diagnostic tools as computed tomographic scan and hemodynamic monitoring, allowing early and reliable evaluation. Worse sensitivities were observed for PE and for respiratory and abdominal causes. This might be explained by more unspecific and atypical clinical symptoms, especially in intubated and sedated patients. In other words, early recognition of abdominal and respiratory complications might be more difficult for cardiac surgeons. 


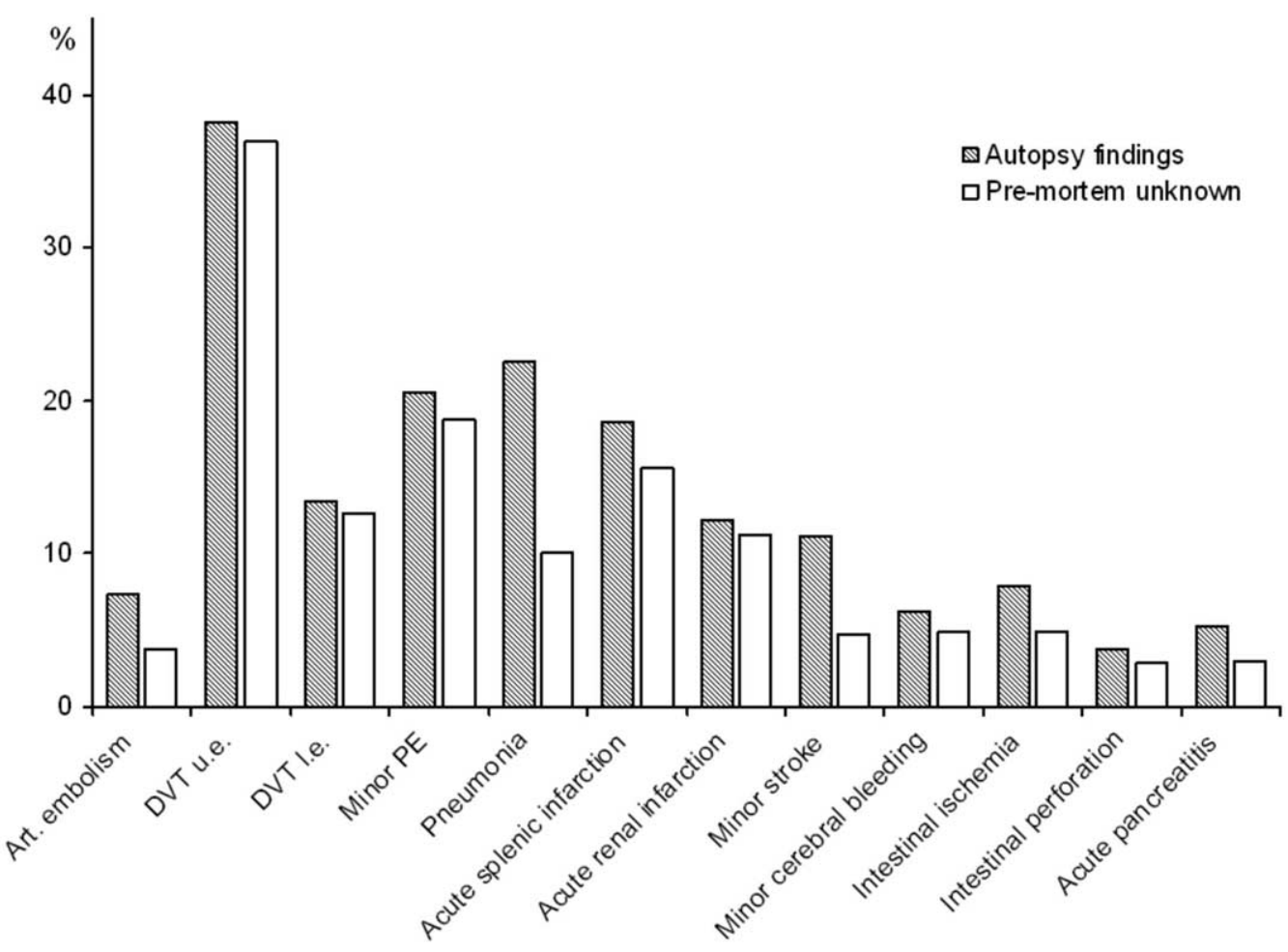

Figure 1. Incidences of perioperative complications as percentages of all patients studied by autopsy. Striped bars represent complications identified by autopsy examination; white bars represent premortem unknown complications. Note high rate of clinically unknown thromboembolic findings. Art., Arterial; u.e., upper extremity; I.e., lower extremity.

According to an organ-related evaluation, the discrepancy rate in our series between clinical and autopsy causes of death was $23.1 \%$; it increased to $33.8 \%$ when using a diagnosis-related analysis. Unexpected causes of death were slightly higher than reported in other series (Table 4). ${ }^{12-16}$ However, the extent of confirmation between clinically assumed and autopsy causes of death was strongly dependent on the underlying causes of death. It varied between $25 \%$ for SCD-a usually ambiguous clinical situation-and $100 \%$ for most cerebral and procedure-associated causes of death.

A major reason for discrepancies between diagnosisrelated clinical and autopsy causes of death was that cardiac failure resulting from AMI could not be clinically identified as a cause of death in a significant number of cases. Thus it can be presumed that surrounding clinical circumstances with further organ dysfunctions make it more difficult for the ICU physician to focus on this cardiovascular event.

AMI was associated with bypass graft dysfunction in 18 patients only. On the other hand, 108 patients had AMI despite good graft function, and 37 had AMI in the absence of significant coronary artery disease. This would suggest that most AMIs stemmed from intraoperative ischemiareperfusion damage.

Regarding PE, literature data support an incidence of $0.4 \%$ to $9.5 \%$ after cardiac surgery, and $0.3 \%$ to $1.7 \%$ of these events are fatal. ${ }^{19,20}$ Because of this rather low incidence, $\mathrm{PE}$ is considered to be an uncommon cause of death for the average patient after cardiac surgery. In autopsy studies of cardiothoracic patients, however, fatal PE is common and has been reported as a cause of death for $4.1 \%$ to $5.5 \%{ }^{13,14,17}$ In our study, this cause of death was unrecognized clinically, representing $11.4 \%$ of all unexpected deaths.

Little is known about patients with SCD after an uneventful postoperative course. ${ }^{16}$ By autopsy, it was possible to clarify the cause of death for $75 \%$ of the 32 patients with SCD. This included acute myocardial infarction in 14 cases. However, in 8 cases no morphologic reason could be identified, and arrhythmia was assumed to be the final cause of death.

Anticoagulation was partial thromboplastin timeadjusted intravenous heparinization (1.5-2 fold) for all patients in the ICU and combined antiplatelet and subcutane- 
TABLE 4. Comparison of previously published series of postmortem examinations in cardiac surgery

\begin{tabular}{|c|c|c|c|c|c|}
\hline & Current study & $\begin{array}{c}\text { Lee and Gallagher, } \\
1997\end{array}$ & $\begin{array}{l}\text { Zehr et al, }^{14} \\
\quad 1997\end{array}$ & $\begin{array}{c}\text { Deiwick et al, } \\
1999\end{array}$ & $\begin{array}{c}\text { Goodwin et al, }{ }^{15} \\
2000\end{array}$ \\
\hline No. of patients & 468 & 108 & 147 & 35 & 167 \\
\hline Country & Germany & UK & US & Germany & UK \\
\hline Autopsy rate (\%) & 52.1 & 87.8 & 24.5 & 46.1 & 84.4 \\
\hline Mean patient age (y) & 68.7 & Unspecified & 60.4 & 68.6 & 69.8 \\
\hline Postoperative survival (d) & 13.9 & $50 \%<1 \mathrm{wk}$ & 22.0 & 11.5 & 7.9 \\
\hline \multicolumn{6}{|l|}{ Autopsy cause of death (\%) } \\
\hline Cardiac & 49.8 & 52.0 & 26.5 & 68.6 & 67.6 \\
\hline Respiratory & 8.3 & 2.4 & 4.1 & 11.4 & 8.4 \\
\hline Cerebral & 6.4 & 5.7 & 8.8 & 5.7 & 3.6 \\
\hline Abdominal & 4.7 & 3.3 & 1.4 & 2.9 & 9.6 \\
\hline MOF & 14.9 & 3.2 & 15.6 & 11.4 & 4.8 \\
\hline $\mathrm{PE}$ & 6.6 & 4.9 & 4.1 & 0 & 0 \\
\hline Procedure associated & 8.3 & 14.6 & 9.5 & 0 & 4.8 \\
\hline Others & 0.9 & 0 & 4.8 & 0 & 1.2 \\
\hline Not clarified & 0 & 13.9 & 25.2 & 0 & 0 \\
\hline $\begin{array}{l}\text { Clinically unexpected } \\
\text { organ-related death (\%) }\end{array}$ & 23.1 & 14.8 & 13.6 & 22.9 & 11.4 \\
\hline
\end{tabular}

ous anticoagulation therapy in others. Despite this regimen, incidence of DVT is about $20 \%$ in the early postoperative course after cardiac surgery, but it was clinically unnoticed in most patients. ${ }^{21}$ Postoperative risk factors for DVT and PE could be clearly identified as immobilization, obesity, recent AMI, hyperlipidemia, coagulation disorders, and central venous catheters. ${ }^{19}$ Taking this into consideration, it is obvious that our patients were at highest risk for DVT formation and PE. We also found arterial emboli in $7.3 \%$ of the patients. Because of the high incidence of acute renal and splenic infarction and the significant number of recognized intracardiac and prosthetic valve thrombi by visualization, we have to conclude that prophylaxis against thromboembolic events remain one of the most challenging fields in the postoperative course after cardiac surgery.

Abdominal complications were identified by autopsy in 80 patients. About two thirds were not clinically recognized. Thus the gastrointestinal tract was of considerable relevance in our study. In particular, for abdominal disorders an early gastroenterologic assessment and consultation of abdominal surgeons might help to identify abdominal disorders earlier and reduce abdominal-associated morbidity and mortality.

We found coronary bypass dysfunction to be the main surgical failure, in $15 \%$ of all patients. In two thirds of these patients $(9.6 \%)$, it was clinically unrecognized. Only 1 autopsy study has focused on that issue; it derived from the venous bypass graft era and reported bypass dysfunction in $29 \%$ after a mean of 153 postoperative days. ${ }^{22}$ However, bleeding, tamponade, and prosthetic valve endocarditis, as well as left ventricle dissection, pericardial tamponade, and aortic rupture, were seen in individual patients and were jointly responsible for surgery-associated mortality in $8.3 \%$ of the cases.

Increasing patient age is associated with an increasing number of severe comorbidities and adverse perioperative outcome. ${ }^{23,24}$ Liver cirrhosis was found in $12.4 \%$ of our patients, with 50\% unknown before death. These severe comorbidities pose additional patient risk, particularly in cardiac surgery and associated transesophageal echocardiography. Liver cirrhosis was unrecognized more frequently in patients with emergency indications, reflecting a special risk to patients without thorough examination. Taking this into consideration, a liberal indication for abdominal ultrasonography, especially for unscheduled patients, has become part of our protocol.

Unknown malignant tumors were recognized by autopsy in $6.2 \%$ of the patients. Presumably only in a minority of cases a more detailed tumor screening would reveal the malignancy and would lead to a reassessment of the individual surgical indication. Nevertheless, this finding underlines the high risk for severe comorbidities in our elderly patient population.

\section{References}

1. Fernandez-Segoviano P, Lazaro A, Esteban A, Rubio JM, Iruretagoyena JR. Autopsy as quality assurance in the intensive care unit. Crit Care Med. 1988;16:683-5.

2. Kircher T, Nelson J, Burdo H. The autopsy as a measure of accuracy of the death certificate. N Engl J Med. 1985;313:1263-9.

3. Barendregt WB, deBoer HH, Kubat K. Autopsy analysis in surgical patients: a basis for clinical audit. Br J Surg. 1992;79:1297-9.

4. Kirch W, Schafii C. Misdiagnosis at a university hospital in 4 medical eras. Report on 400 cases. Medicine. 1996;75:29-40. 
5. Goldman L, Sayson R, Robbins S, Cohn L, Bettman M, Weisberg M. The value of autopsy in three medical eras. N Engl J Med. 1986;28: 1000-5.

6. Grundmann E. Autopsy as clinical quality control: a study of 15.143 autopsy cases. In Vivo. 1994;8:945-52.

7. Mort TC, Yeston NS. The relationship of premortem diagnoses and postmortem findings in a surgical intensive care unit. Crit Care Med. 1999;27:299-303.

8. American College of Chest Physicians/Society of Critical Care Medicine Consensus Conference: definition of sepsis and organ failure and guidelines for the use of innovative therapies in sepsis. Crit Care Med. 1992;20:864-74.

9. Sonderegger-Iseli K, Burger S, Muntwyler J, Salomon F. Diagnostic errors in three medical eras: a necropsy study. Lancet. 2000;355:2027-31.

10. Lee PN. Comparison of autopsy, clinical and death certificate diagnosis with particular reference to lung cancer. A review of the published data. APMIS Suppl. 1994;45:1-42.

11. Battle RM, Pathak D, Humble CG, Key CR, Vanatta PR, Hill RB, et al. Factors influencing discrepancies between premortem and postmortem diagnoses. JAMA. 1987;258:339-44.

12. Deiwick M, Löhrer A, Hoffmeier A, Baba HA, Böcker W, Scheld HH. Postoperative death should be followed by autopsy-an analysis of the autopsy findings of the years 1990 and 1991 in a heart surgery center. Thorac Cardiovasc Surg. 1999;47:82-7

13. Lee AH, Gallagher PJ. Post-mortem examination after cardiac surgery. Histopathology. 1998;33:399-405.

14. Zehr KJ, Liddicoat JR, Salazar JD, Gillinov AM, Hruban RH, Hutchins MH, et al. The autopsy: still important in cardiac surgery. Ann Thorac Surg. 1997;64:380-3.

15. Goodwin AT, Goddard M, Taylor GJ, Ritchie AJ. Clinical versus actual outcome in cardiac surgery: a post-mortem study. Eur J Cardiothorac Surg. 2000;17:747-51.

16. Lee AH, Borek BT, Gallagher PJ, Saunders R, Lamb RK, Liversey SA, et al. Prospective study of the value of necropsy examination in early death after cardiac surgery. Heart. 1997;78:34-8.

17. Ooi A, Goodwin AT, Goddard M, Ritchie AJ. Clinical outcome versus post-mortem findings in thoracic surgery: a 10-year experience. Eur J Cardiothorac Surg. 2003;23:878-81.

18. Boldy DA, Jones C, Matthews H, Edwards C. Death and necropsies in a thoracic unit. Thorax. 1993;48:284-6.

19. Shammas NW. Pulmonary embolus after coronary artery bypass surgery: a review of the literature. Clin Cardiol. 2000;23:637-44.

20. Josa M, Siouffi SY, Silverman AB, Barsamian EM, Khuri SF, Shar GV. Pulmonary embolism after cardiac surgery. J Am Coll Cardiol. 1993;15:990-6.

21. Goldhaber SZ, Hirsh DR, MacDougall RC, Polak JF, Creager MA, Cohn LH. Prevention of venous thrombosis after coronary artery bypass surgery. Am J Cardiol. 1995;76:993-6.

22. Rose AG. State of the vein grafts, native coronary arteries, and myocardium and principal cause of death in patients dying after aortocoronary artery bypass grafting. Thorax. 1985;40:940-7.

23. Warner CD, Weintraub WS, Craver JM, Jones EL, Gott JP, Guyton RA. Effect of cardiac surgery patient characteristics on patient outcomes from 1981 through 1995. Circulation. 1997;96:1575-9.

24. Smith CJ, Scott SM, Wagner BM. The necessary role of the autopsy in cardiovascular epidemiology. Hum Pathol. 1998;29:1469-79.

\section{Discussion}

Dr Edward Verrier (Seattle, Wash). This is a remarkable study, as it must be because we are looking at autopsies, of 16,464 operations in 14,313 patients undergoing elective, urgent, and emergency cardiac procedures during a relatively short concurrent 4-year period. In all, 898 patients, died for an overall mortality of $6.3 \%$, which was constant through the 4 -year period. I believe the results represent an outstanding clinical experience from a single institution. The autopsy rate was $52 \%$, which is also remarkable in an era when overall postmortem examination rates have decreased, the most obvious reason being the fear of medicolegal recrimina- tions. The article contains a huge amount of information, much of which is relevant and useful because of the large cohort size and the careful analysis. I encourage all cardiac surgeons to read the published article.

The overall correlation between the clinical assessment and the autopsy confirmation of death was $76 \%$. One of the most striking observations was the high confirmation rates between clinical assessment and autopsy for cardiac causes (85\%), cerebral causes (86\%), procedure-related causes $(96 \%)$, and fatal PE (81\%). In contrast, the confirmation rates for respiratory distress and pneumonia (54\%), abdominal causes (65\%), and MOF (66\%) were much lower.

My first question, Dr Rastan, is what have you learned from these observations, and how has it affected your clinical practice prospectively? Are there better yet cost-effective ways to assess the lungs or abdomen either preoperatively or perioperatively that would lead either to more aggressive diagnostic definition or to alternative therapeutic strategies? Are there patient cohorts with we should be more aggressive in using chest or abdominal computed tomography, magnetic resonance imaging, noninvasive vascular testing, or assay of systemic markers of inflammation? We already have literature stating that extensive screening tests are often not cost-effective without symptoms. We also know that broad-spectrum antibiotic use or aggressive anticoagulation regimens are double-edged swords in terms of risk-benefit ratio. What are your thoughts?

Dr Rastan. Thank you, Dr Verrier, for your comments. Thinking about consequences, we have to find individual therapeutic strategies for elderly patients, bearing in mind that a third of our study population $(\mathrm{n}=284)$ were 75 years or older. This includes asking patients at risk to come to our surgical outpatient department for evaluation of their cardiac symptoms, comorbidities, and cerebral status and discussing their individual surgical risk. Therapeutic options could include off-pump CABG procedures and hybrid minimally invasive direct $\mathrm{CABG}$ procedures or asking the cardiologists for percutaneous coronary intervention, being aware that we would achieve only incomplete revascularization in individual patients. However, the more we decline to operate on patients in critical condition, the more we can reduce mortality. Therefore mortality could be significantly reduced by refusing to operate on patients with cardiogenic shock, for example, which we accept liberally at the moment.

Another point is the timing of operation, bearing in mind that overall mortality increased to $9.3 \%$ for urgent indications and $19.4 \%$ for emergency indications. In selected patients with acute coronary symptoms, a delayed operation could be of benefit by allowing myocardial recovery, accurate preoperative preparation, and diagnostic evaluation.

A further consequence is to reflect about preoperative diagnostics. I agree with you that nonspecific computed tomography and magnetic resonance imagery will not be helpful in most cases. For patients at risk, however, we have changed our protocol and use ultrasonography, computed tomographic scan, and even nuclear magnetic resonance more liberally, especially in emergency cases.

Additionally, we should encourage our cardiologists to carry out a detailed preoperative medical evaluation of surgical candidates, including abdominal ultrasonography. This could help to re- 
duce the numbers of patient with severe unknown comorbidities, such as malignancy, and to reduce unfavorable postoperative outcomes.

As I mentioned, our protocol includes an individual concept of specific intravenous antibiotic therapy in every ICU patient. It is our consensus that a more aggressive nonspecific treatment is not appropriate.

Dr Verrier. There are certainly groups that have discrepancies. For instance, you note a higher than expected discrepancy between clinical and autopsy causes of death because congestive heart failure was often not identified clinically as a problem leading to death.

What is the best way, for instance, to recognize subtleties of postoperative heart failure? Longer hemodynamic monitoring, more widespread use of myocardial enzymes as markers of injury? Should we be keeping our marginal patients on inotropics longer? Should we be more aggressive with the use of angiotensin-converting enzyme inhibitors, selective $\beta$-blockade, or natriuretic hormone therapy, or more liberal with consultations? Have these types of things come into the way you have changed your practice since you have examined these data so carefully?

Dr Rastan. A total of 233 patients had autopsy-confirmed cardiac causes of death. In 186 cases, cardiac causes of death were clinically assumed. In the others, clinically assessed causes of death were predominantly pneumonia and MOF. Most of these patients had prolonged postoperative course of more than 2 weeks. So we can conclude that lingering cardiac failure with and without AMI still occurs weeks after the operation. Second is that in the later postoperative course cardiac failure seems to be not as obvious as in the early postoperative period. Therefore one lesson is to think of cardiac failure in these patients and to treat it as early as possible by medical or mechanical means. Patients with remission of early postoperative heart failure are especially at risk and should be carefully monitored for a prolonged time with optimized heart failure medication, including inotropics and intra-aortic balloon pump when appropriate.

We are monitoring the cardiac enzymes carefully, and in every case in which we suspect bypass dysfunction, we take the patient back to the catheterization laboratory for angiography. Interestingly, more than $90 \%$ of the patients with signs of AMI had excellent bypass function. We found that AMI was more frequent in patients with coronary artery disease, but it was not strongly associated with bypass dysfunction in these patients. Not surprisingly, it was evident that patients with combined procedures and prolonged myocardial ischemia were at risk for AMI. So we have to think about myocardial protection in the operating room, avoiding long ischemia and doing beating-heart surgery in critical cases, including patients with acute coronary syndrome.

Dr Verrier. The final striking observation is this very high incidence of pathologic findings found perioperatively, $77 \%$ of the patients, most of which weren't known until post mortem, and these include arterial emboli, phlebothrombosis, minor PE, pneumonia, splenic and renal infarction, minor cerebral events, intestinal ischemia or perforation, and pancreatitis. In addition, a total of 928 concomitant diagnoses were identified, of which only 464 had been identified postmortem, such as left ventricular hypertrophy, occlusive arterial disease, pulmonary emphysema, cirrhosis, and malignancy. Of these conditions, you state that almost $20 \%$ had surgical implications, $14 \%$ in the emergency group and 5\% in the elective group. If known, would the operations have not been done, or would they have been better delayed, and if delayed, would the knowledge have made a difference in the overall impact?

I guess the bottom line is this: how can we improve on the numbers to actually benefit this sick group of patients, rather than simply benefiting the statistics? I thank the Association for the privilege of discussing this important article. Postmortem examination after cardiac surgery must remain a part of our quality assurance programs.

Dr Rastan. The high rates of unknown concomitant diseases were unexpected for us. In addition to unknown comorbidities of minor operative impact, we also found severe comorbidities in $20 \%$, especially more than $6 \%$ of unknown malignancy. The knowledge of severe comorbidities is a condition for successful surgery and more or less influences the surgical indication and operative strategy. However, it is difficult to speculate about the individual dimension.

Regarding the postoperative complications, I just want to focus on the surprisingly high incidence of thromboembolic events we found in the venous system but also in the arterial system. Even though all patients received adjusted intravenous anticoagulation with heparin, we found an incidence of minor PE of $20 \%$. Interestingly, most of these events were clinically completely unrecognized. This makes us aware that the anticoagulation regimen is one of the major challenges for ICU patients, because our population is a subset of patients with the highest risk for perioperative morbidity and mortality.

Dr Craig Smith (New York, NY). I think this is potentially very important information, and you are to be congratulated on obtaining a $52 \%$ autopsy rate, certainly far better than we achieve. It would help me understand the clinical diagnoses you discuss if you could express them, as we do for modalities like computed tomographic scan or magnetic resonance imaging, in terms of sensitivity and specificity. I'm particularly interested in one diagnosis that we frequently consider in this setting but seldom confirm at autopsy, and that is PE. Can you tell me your sensitivity and specificity for the clinical diagnosis of PE?

Dr Rastan. Thank you, Dr Smith, for your question. In 16 patients of our cohort, fatal PE was clinically assumed as cause of death. In 13 of these patients, PE was confirmed by autopsy, leading to a confirmation or positive predictive value of this clinical cause of death of $81.3 \%$. On the other hand, by autopsy fatal $\mathrm{PE}$ was found in 31 patients. Thus, the clinical sensitivity for this cause of death was only $41.9 \%$. So we have to keep this diagnosis in mind.

Dr Mohr. Maybe I can have a final comment in terms of what clinical impact it has, and the problem arising in the emergency situation. We do have the situation that if we get the patients late at night in the operating room, they have incomplete diagnoses. If you look into patients with endocarditis, which occurs quite often, they present with splenic emboli and so on. Now we insist on a quick total body preoperative computed tomographic scan on these patients, and if something is there, it is going to be solved in a one-stage operation; we go for a one-stage splenectomy right away, for example, as one of the situations. Also, the computed tomographic scan helps to identify a mesenteric ischemia in some patients preoperatively. So if we do see that, we have to consider whether we should keep our hands off these patients. 